\title{
Predicting microwave-induced relative volatility change by a novel dimensionless number
}

\author{
Zhenyu Zhao ${ }^{1}$, Hong $\mathrm{Li}^{2}$, Guanlun Sun ${ }^{1}$, Xinhui Tang ${ }^{3}$, Haoyu $\mathrm{Wu}^{1}$, and Xin $\mathrm{Gao}^{1}$ \\ ${ }^{1}$ Tianjin university \\ ${ }^{2}$ National Engineering Research Ceter of Distillation Technology \\ ${ }^{3}$ Affiliation not available
}

November 22, 2020

\begin{abstract}
Microwave (MW) can selectively interact with polar molecules in homogeneous mixture liquid solution and therefore change their relative volatility, which has been previously observed in experimental results for various binary mixtures. However, the lack of theoretical analysis and quantitative modeling hinders the further development of novel MW-assisted separation techniques. Hence, this study derived a novel dimensionless number ZMW based on the assumption of molecular irradiation to explore the effect of the dielectric and thermodynamic properties of materials as well as MW field intensity on the microwave-induced relative volatility change (MIRVC). Furthermore, a quantitative correlation between MIRVC and ZMW was established, whose model parameters were determined by fitting experimental data under MW irradiation. The correlation was also utilized to predict MIRVC in the previously published literature and the error range between predictive values and experimental values was within $\pm 6 \%$, indicating great validity of the proposed quantitative correlation.
\end{abstract}

\section{Hosted file}

Manuscript.pdf available at https://authorea.com/users/377856/articles/494513-predictingmicrowave-induced-relative-volatility-change-by-a-novel-dimensionless-number 